\title{
AN INTRODUCTION TO THE CLASSIFICATION OF FINITE SIMPLE GROUPS
}

\author{
BY MICHAEL ASCHBACHER
}

The classification of the finite simple groups now seems close at hand. You may judge for yourself just how close from Daniel Gorenstein's article in this Bulletin, introduced below.

One of the most important techniques in modern science and mathematics is the investigation of an object from the point of view of its symmetry group. This approach originated in Galois' investigation of number fields, which lead to a proof of the insolvability of the quintic. The groups considered by Galois were finite, as are the groups arising in many other interesting problems. This is fortunate as finite groups are much more tractable than infinite groups. For example it is difficult to imagine that a classification of the infinite simple groups will ever be achieved.

The axioms describing finite groups are concise and natural. They lead to a truly rich structure represented by a large and satisfying family of examples. The finite simple groups are particularly interesting. The groups of Lie type are the finite analogues of the semisimple algebraic and Lie groups. Following Tits, the groups of Lie type may be defined as the finite groups $G$ with a $(B, N)$-pair, where $B$ is the normalizer of some Sylow group of $G$ which is a complement to $B \cap N$ in $B$. Consider for example the group $G L(n, F)$ of $n$ by $n$ nonsingular matrices over $F$. If $F$ is the complex numbers, $G L(n, F)$ is a Lie group. If $F$ is a finite field, $G L(n, F)$ is a group of Lie type. The groups of Lie type come equipped with an important geometric structure, the Tits building. The building of $G L(n, F)$ is the simplicial complex whose points are the collection of subspaces of an $n$-dimensional vector space over $F$, with simplices defined by inclusion. While each semisimple Lie group has its analogue among the finite simple groups, the converse is not true. In addition to the ordinary groups of Lie type there are certain twisted groups of Lie type with no continuous analogue, there are the groups of prime order, the alternating groups, and finally there are twenty-four further finite simple groups, together with overwhelming evidence for the existence of yet two more. The last twenty-six groups are called the sporadic groups. Many mathematicians other than simple group theorists find them fascinating.

Of course the simple groups are the building blocks of the finite groups. Determining the simple groups and their internal properties leave many questions about finite groups unanswered but makes possible the solution of many others. I mention only three examples, each over half a century old. The first is a conjecture of Schreier that the outer automorphism group of a

Received by the editors May 1, 1978.

AMS (MOS) subject classifications (1970). Primary 20D05.

() American Mathematical Society 1979 0002-9904/79/0000-0004/\$01.75 
simple group is solvable. The second is a conjecture of Frobenius that if $n$ is a divisor of the order of a finite group $G$ in which there are exactly $n$ solutions to the equation $x^{n}=1$, then these solutions form a subgroup of $G$. The final problem is to determine all multiply transitive permutation groups. A permutation group $G$ on a set $X$ is $n$-transitive if any pair of ordered $n$-tuples of distinct elements of $X$ may be mapped into one another by a permutation in $G$. Modulo some properties of the known simple groups, most of which are already established, each problem would admit a solution if the present list of simple groups were shown to be complete. Probably none of them could be solved more directly.

In order to classify the simple groups it seems necessary to study finite groups in general. Thus the investigation of finite simple groups has generated a wealth of techniques for studying the general finite group. The resulting theory is complex and sophisticated. It includes Brauer's theory of modular representations, Fischer's internal geometric theory of finite groups, and the local theory of John Thompson, Gorenstein, and others. It is a theory of finite groups not just finite simple groups.

Brauer's theory has been profitably applied in a number of areas of mathematics. The other techniques seem to have remained within the province of the simple group theorist. This may be because the techniques are too specialized to be of use elsewhere, or it may be because no serious attempt has been made to extend them beyond the area of finite groups. If the latter is the case possibly someone reading Gorenstein's article will be led to rectify the situation.

Gorenstein's manuscript is a description of the classification intended for the nonspecialist. It is more or less self contained, and describes in some detail the major steps in the program and the theory developed to deal with them. The article comes in two parts, the first part appearing in this issue of the Bulletin. If the classification is completed as expected the proof will be exceptionally long (Gorenstein estimates over 5,000 pages) and will include the work of many mathematicians. The length of the program is reflected in the length of the article. If you feel reluctant to involve yourself in a manuscript of such length, read as a start just Chapter I. That chapter attempts to give only a general idea of the techniques and proof. Chapter II contains a description of the known finite simple groups, including a history of the discovery of the sporadic groups and details of the machine construction of some of these groups. You might wish to read for example about Fischer's Monster, the largest sporadic group of order

$$
2^{46} \cdot 3^{20} \cdot 5^{9} \cdot 7^{6} \cdot 11^{2} \cdot 13^{3} \cdot 17 \cdot 19 \cdot 23 \cdot 29 \cdot 31 \cdot 41 \cdot 47 \cdot 59 \cdot 71
$$

or about $10^{54}$. Actually this group has not been shown to exist, but two other sporadic groups do exist which were discovered as subgroups of the Monster.

A. Ogg has observed that the set of prime divisors of the order of the Monster is exactly the set of prime divisors arising in a certain question about the super singularity of the reduction modulo $p$ of the modular curve $X_{0}(p)$. He offers a bottle of Jack Daniels to the person explaining this coincidence.

The remaining two chapters in part one discuss portions of the general 
theory of finite groups. For example there is a discussion of signalizer functors. Gorenstein has suggested that some extension of this concept might be of use in investigating rings.

In Chapter I a basic four part subdivision of the classification is indicated. The second part of the article consists of four chapters discussing in detail each part of the subdivision.

This then is an outline of the Gorenstein article. You may decide to read only part of it. I hope however that you will read the entire article and come to better appreciate the fascinating and venerable problem it discusses.

Department of Mathematics, California Institute of Technology, Pasadena, CaliFORNIA 91125 
